\title{
Report of a Case of Primary Signet-ring Cell Carcinoma of the Bladder and Review of the Literature
}

\author{
Baiyun Gao, Guo Chen, Yutong Li, Bin Pan* \\ Department of Urology, The First Affiliated Hospital, Jinan University, Guangzhou, China \\ Email address: \\ 281463483@qq.com (Baiyun Gao),387224538@qq.com (Guo Chen), liyutong@jnu.edu.cn (Yutong Li), panbin@jnu.edu.cn (Bin Pan) \\ ${ }^{*}$ Corresponding author
}

To cite this article:

Baiyun Gao, Guo Chen, Yutong Li, Bin Pan. Report of a Case of Primary Signet-ring Cell Carcinoma of the Bladder and Review of the Literature. International Journal of Clinical Urology. Vol. 4, No. 2, 2020, pp. 92-95. doi: 10.11648/j.ijcu.20200402.23

Received: December 4, 2020; Accepted: December 14, 2020; Published: December 22, 2020

\begin{abstract}
Primary signet-ring cell carcinoma of the bladder is a relatively rare tumor that progresses rapidly and has an extremely poor prognosis. We report a case of primary signet-ring cell carcinoma of the bladder. The patient underwent transurethral holmium laser lithotripsy for bladder calculi in another hospital, and a bladder tumor was found intraoperatively. Bladder tumor biopsy revealed infiltrating high-grade urothelial carcinoma with adenoid differentiation and invasion to the muscular layer. Gastrointestinal endoscopy ruled out other possible sources of tumor, and the patient was admitted to our hospital for treatment in October 2018. Cystoscopy revealed a large round mass in the trigone and right bladder wall, without probing the pedicle of the mass. After radical cystectomy, postoperative pathology showed signet-ring cell carcinoma, which infiltrated the full-thickness of the bladder wall, and cancer emboli were found in vasculature. The prostate gland and bilateral seminal vesicle were involved. After surgery, the patient did not receive any follow-up treatment due to personal factors. The Computed Tomography (CT) review at the 3rd month showed no tumor recurrence or metastasis, and the CEA review at the 6th month was $6790.2 \mathrm{ng} / \mathrm{ml}$, considering that tumor metastasis had occurred. The patient was followed up for 9 months after surgery, and the postoperative survival time was more than 8 months. The prognosis of primary signet-ring cell carcinoma of the bladder is poor and most patients are in advanced stage at the time of diagnosis. Chemotherapy and radiotherapy have poor effect on it. Usually only total cystectomy may bring some hope to patients. Thus, early diagnosis and treatment are crucial.
\end{abstract}

Keywords: Signet-ring Cell Carcinoma, Bladder, Urothelial Carcinoma, Therapy, Prognosis

\section{Introduction}

Primary signet-ring cell carcinoma of the urinary bladder (PBSRCC) is a special type of bladder urothelial carcinoma (UC) [1]. Although bladder urothelial carcinoma accounts for more than $90 \%$ of bladder cancer types, primary signet-ring cell carcinoma of the bladder is still relatively rare. The latest study showed that only 318 of 57,762 patients with urothelial carcinoma had primary signet-ring cell carcinoma, with an incidence of $0.55 \%$ [2]. It is difficult to treat primary signet-ring cell carcinoma of the bladder, which is insensitive to chemotherapy and radiotherapy. Most of them are treated by surgery. Among the different surgical treatments, patients benefited more from radical cystectomy than partial cystectomy with reserved bladder, and cystectomy was closely associated with improved survival rate in patients with primary signet-ring cell carcinoma of the bladder [3]. However, primary signet-ring cell carcinoma of the bladder is an independent predictor of lower overall survival after radical cystectomy compared with pure urothelial carcinoma of the bladder [4]. Signet-ring cell carcinoma of the bladder also showed the worst survival outcome in the histologic subtype of bladder cancer in the SEER database [5]. Unfortunately, the rarity of Primary signet-ring cell carcinoma of the bladder makes it difficult to define the optimal treatment strategy [6]. Here, we report a case of primary signet-ring cell carcinoma of the bladder in a man admitted to our department in October 2018, who underwent radical cystectomy and died of recurrent metastases at the ninth month of postoperative follow-up. 


\section{Case Report}

A 49-year-old man was admitted to another hospital more than 3 months after double nephrostomy due to the discovery of hydronephrosis. On September 17, 2018, the patient underwent transurethral holmium laser lithotripsy for bladder calculi in another hospital, and a bladder tumor was found intraoperatively. Bladder tumor biopsy revealed infiltrating high-grade urothelial carcinoma with adenoid differentiation and invasion to the muscular layer. On September 24, 2018, abdominal CT showed bilateral lower ureteral calculi and dense shadow of posterior bladder wall. The patient was admitted to our department on October 8, 2018 after the detection of bladder cancer for more than a week. The patient had no history of hematuria or abdominal mass. Physical examination revealed an old surgical scar about $8 \mathrm{~cm}$ long in the middle of the upper abdomen and retention of nephrostomy tubes in both kidneys. The patient also had a history of subtotal gastrectomy and cholecystectomy for duodenal bulbar ulcer with hemorrhage on May 22, 2018, but had no abnormalities in postoperative pathology. Because of hydronephrosis, the patient had a history of double nephrostomy as well as long-term history of heavy drinking and smoking. Gastrointestinal endoscopy on September 26,

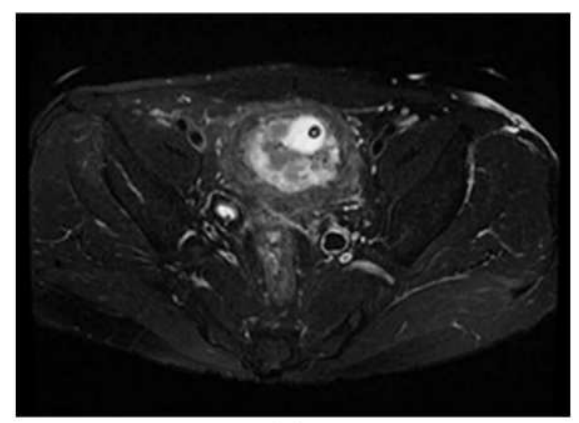

(a)
2018 indicated anastomosis and post-subtotal gastrectomy.

After admission, the tumor markers such as alpha-fetoprotein (AFP), CA19-9 were within the normal range. Carcinoembryonic antigen (CEA) was at $138 \mathrm{ng} / \mathrm{ml}$ while the normal range of it should be under $5 \mathrm{ng} / \mathrm{ml}(<5 \mathrm{ng} / \mathrm{ml})$. Urine routine indicated that urine occult blood positive. Pelvis magnetic resonance (MR) showed the bladder wall was unevenly thickened and an about $6.9 \mathrm{~cm} \times 2.9 \mathrm{~cm}$ soft tissue mass was visible with heterogeneous intensity, $\mathrm{T} 1$ weighted imaging (T1W1) with hypointensity, T2 fat suppression (T2 fs) with hyperintensity, Diffusion Weighted Imaging (DWI) with hyperintensity, and the enhancement scan showed uneven and obvious intensification. A saccule of about $0.6 \mathrm{~cm}$ in diameter protruded from the right wall of the bladder. There was bilateral lower ureteral dilatation in which calculi shadow with hypointensity could be seen, with one on the left side with a size of approximately $0.9 \mathrm{~cm} \times 1.0 \mathrm{~cm} \times 1.3 \mathrm{~cm}$, and two on the right with a size of approximately $1.0 \mathrm{~cm} \mathrm{x} 1.1 \mathrm{~cm} \times 1.1 \mathrm{~cm}$ and $0.3 \mathrm{x}$ $0.3 \times 0.2 \mathrm{~cm}$. The volume of prostate was normal with heterogeneous intensity and no obvious abnormal enhancement was found in the enhancement scan (Figure 1). Cystoscopy revealed a large round mass in the trigone and right bladder wall, without probing the pedicle of the mass.

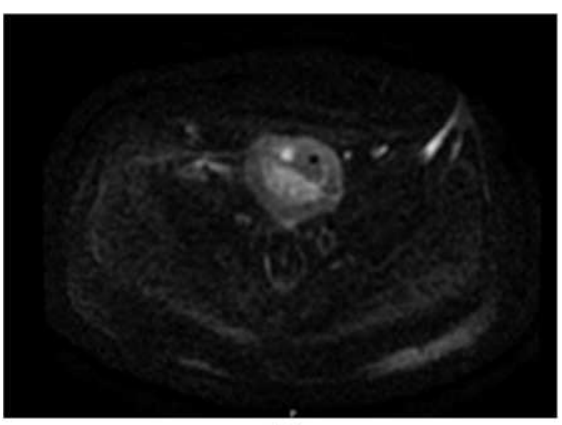

(b)

Figure 1. MR: the bladder wall was unevenly thickened and a soft tissue mass was visible. (T2 fs with hyperintensity (a), DWI with hyperintensity (b).

Considering that radical cystectomy did better to patient's survival, radical cystectomy was performed under general anesthesia. An incision was made in the center of the abdomen, away from the bladder, seminal vesicles and the tip of the prostate. Both ureters were cut off, and adhesions between the prostate and Denonvilliers' fascia and mucosal tumors of the lower segment of both ureter were observed. Postoperative incision of the bladder revealed multiple irregular masses in the bladder (Figure 2). Postoperative pathology: signet-ring cell carcinoma, which infiltrated the full-thickness of the bladder wall, and cancer emboli were found in vessels, involving the prostate gland and bilateral seminal vesicle. There was no carcinoma in prostate urethral margins, ureteral margins on both sides, and spermaduct margins. Immunohistochemistry: CK7(-), CK20(+), CDX-2(+), Villin (+), GATA-3(-), P53(-), Ki- 67 approximately 80\% (+) (Figure 3). Diagnosis: primary signet-ring cell carcinoma of the bladder (pT4aNxM0). After surgery, the patient did not receive any follow-up treatment due to personal factors. The $\mathrm{CT}$ review at the $3^{\text {rd }}$ month showed no tumor recurrence or metastasis, and the CEA review at the $6^{\text {th }}$ month was 6790.2 $\mathrm{ng} / \mathrm{ml}$. Considering the occurrence of pernicious anemia, tumor metastasis might have occurred. The patient was followed up for 9 months after surgery, and his postoperative survival was more than 8 months.

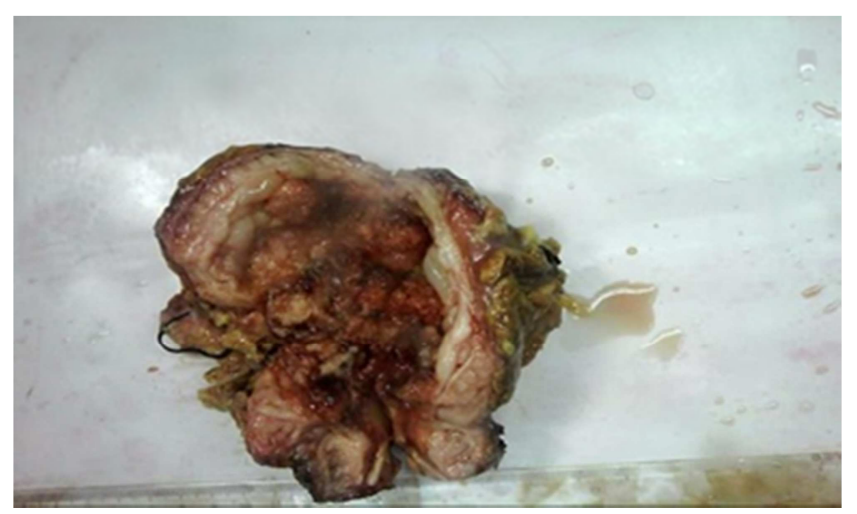

Figure 2. After the bladder was cut open, multiple irregular masses were found in the bladder. 


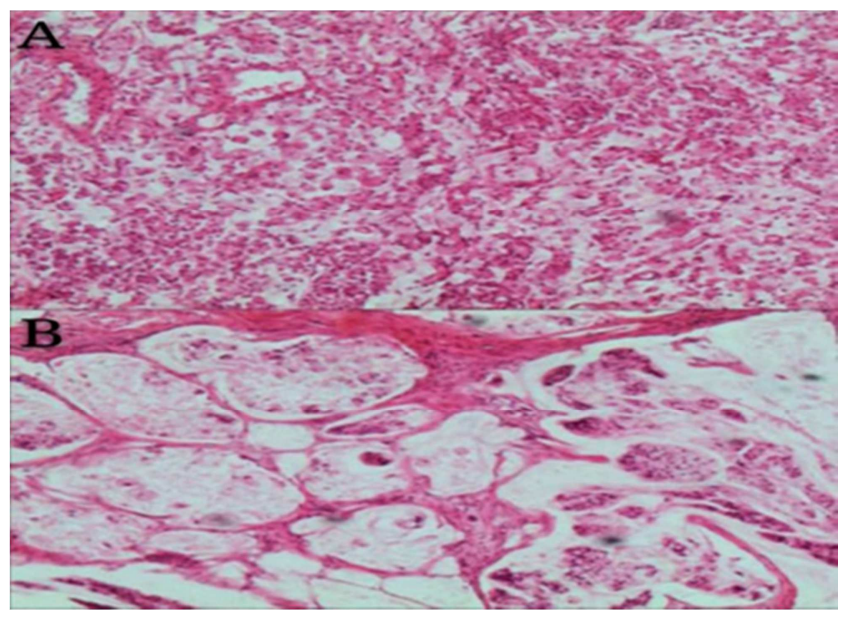

Figure 3. (A) Signet cell carcinoma invading the submucosal tissue (H and E $\times 100)$; (B) Signet cells with pushed-out hyperchromatic nuclei (H and E, $\times 400)$.

\section{Discussion}

Primary signet-ring cell carcinoma of the bladder usually occurs in patients at the age of approximately 57-76 years old and mainly in males, with a male to female ratio of approximately 3:1 [2]. The clinical manifestations of primary signet-ring cell carcinoma of the bladder are not significantly different from other types of bladder cancer, and the main symptoms are urinary irritation and hematuria [3,7], so it is difficult to distinguish primary signet-ring cell carcinoma of the bladder according to the clinical manifestations alone. Primary signet-ring cell carcinoma of the bladder can spread laterally and widely without protruding through the surface, most of which spread as infiltrating growth rather than the exogenous growth, which is not easy to identify in cystoscopy. Most patients did not have mucosal lesions or mass on cystoscopy. Instead, they only had mucosal edema [8]. This causes that it cannot be detected and diagnosed early. The cystoscopy biopsy may also be missed, and a definitive diagnosis may require a full-thickness bladder biopsy [9]. In some patients, obstruction and hydronephrosis may occur during the course of the disease due to diffuse infiltrative lesions compressing the ureter [10]. In the current case, bilateral hydronephrosis occurred in the early stage, and bilateral nephrostomy was performed for symptomatic treatment. Urine routines showed visible microscopic hematuria. Cystoscopy revealed a large round mass in the trigone and right bladder wall, without probing the pedicle of the mass, and bilateral ureteral openings were not found. CT showed dense shadow of posterior bladder wall. It was predicted that the tumor had invaded the lower ureter segment and compressed bilateral ureteral openings, resulting in hydronephrosis. Cystoscope biopsy revealed urothelial carcinoma with adenoid differentiation, and full-thickness bladder pathology revealed signet-ring cell carcinoma.

Signet-ring cell carcinoma frequently appears in the gastrointestinal tract [11], and the diagnosis of primary signet-ring cell carcinoma of the bladder should first exclude metastatic tumor of gastrointestinal origin. Studies have shown that CK7 was a useful markers for metastatic signet ring cell carcinoma of gastric and colorectal origin (with $93 \%$ sensitivity and 92\% specificity). CK20 and CDX2 had very limited effect on delineating the primary sites of metastatic signet-ring cell carcinomas of gastrointestinal origin [12]. Bladder cancer consisting solely of signet-ring cells should be considered as a distant metastasis, while tumors consisting of a mixture of signet-ring cells and urothelial cells should be considered as primary bladder cancer [13]. This case showed CK7(-), CK20(+), CDX-2(+), and metastatic tumor of gastrointestinal origin was excluded by pathology and gastrointestinal endoscope. There were both urothelial cells and signet-ring cells in pathology.

Radical cystectomy is more preferred to be the first choice as for the treatment for primary signet-ring cell carcinoma at present [14]. Chemotherapy and radiotherapy show no good effect on it [15]. It remains unclear whether chemotherapy and (or) radiotherapy combined with surgery can improve the prognosis. Some scholars abroad reported that adjuvant chemotherapy with cisplatin in combination with 5-fluorouracil can improve OS for signet-ring cell carcinoma [16]. In the present case, the patient did not receive subsequent chemotherapy due to personal factors. As for the prognosis of primary signet-ring cell carcinoma, previous studies have shown that elevated CEA levels may be an indicator of poor prognosis in primary signet-ring cell carcinoma of the bladder [8]. The elevation is negatively correlated with the prognosis of primary signet-ring cell carcinoma of the bladder. The CEA of this patient was $138 \mathrm{ng} / \mathrm{ml}$ for the first time and 6790.2 $\mathrm{ng} / \mathrm{ml}$ for the reexamination at $6^{\text {th }}$ month after the operation, which suggested a poor prognosis.

\section{Conclusion}

In the current case, the clinical symptoms were not obvious, and hydronephrosis occurred in the early stage. The early diagnosis was incomplete due to the combination of bilateral ureteral stones. When the patient was first found to have a tumor, it was already advanced bladder cancer and had invaded the lower part of the ureter. Taking into consideration the patient's physical condition, in order to reduce the tumor and for palliative purpose, radical cystectomy was performed under general anesthesia instead of reconstruction of bladder with ileum. Postoperative pathology reported no carcinoma in prostate urethral margins, ureteral margins on both sides and vas deferens margins. Due to the patient's personal factors, no follow-up treatment was performed, so the therapeutic effect of chemotherapy and radiotherapy on the patient could not be evaluated. The postoperative survival time of the patient was more than 8 months. As for how to conduct early diagnosis for this rare disease, it still needs further exploration. In order to avoid incomplete diagnosis, cystoscopy and biopsy can be considered in the early detection of hydronephrosis to determine whether it is related to bladder lesions. How to improve the survival rate of primary signet-ring cell carcinoma of the bladder still needs a lot of clinical research. 


\section{Acknowledgements}

This work was supported by the Leading Specialist Construction Project-Department of Urology, the First Affiliated Hospital, Jinan University (711006).

\section{References}

[1] Humphrey PA, Moch H, Cubilla AL, Ulbright TM, Reuter VE. (2016). The 2016 WHO Classification of Tumours of the Urinary System and Male Genital Organs-Part B: Prostate and Bladder Tumours. European urology 70 (1): 106-19.

[2] D J, S Q, K J, X Z, Q C, L Y, et al. (2020). Signet-Ring Cell Carcinoma as an Independent Prognostic Factor for Patients With. Front Oncol 2020 May 15; 10: 653.

[3] Wang J, Wang FW. (2011). Clinical Characteristics and Outcomes of Patients with Primary Signet-Ring Cell Carcinoma of the Urinary Bladder. Urologia Internationalis 86 (4): 453-60

[4] Veskimäe E, Espinos EL, Bruins HM, Yuan Y, Sylvester R, Kamat AM, et al. (2019). What Is the Prognostic and Clinical Importance of Urothelial and Nonurothelial Histological Variants of Bladder Cancer in Predicting Oncological Outcomes in Patients with Muscle-invasive and Metastatic Bladder Cancer? A European Association of Urology Muscle Invasive and Metastatic Bladder Cancer Guidelines Panel Systematic Review. European urology oncology 2 (6): 625-42.

[5] Natale C, Leinwand GZ, Chiang J, Silberstein JL, Krane LS. (2019). Reviewing the Demographic, Prognostic, and Treatment Factors of Primary Adenocarcinoma of the Bladder: A SEER Population-based Study. Clinical genitourinary cancer 17 (5): $380-8$.

[6] Palsgrove DN, Taheri D, Springer SU, Cowan M, Guner G, Mendoza Rodriguez MA, et al. (2019) Targeted sequencing of plasmacytoid urothelial carcinoma reveals frequent TERT promoter mutations. Hum Pathol 85: 1-9.

[7] Bouhajja L, Farah F, Garbouj N, Rammeh S. (2019). Primary signet-ring cell carcinoma of the urinary bladder: A report of two cases. La Tunisie medicale 97 (1): 167-9.
[8] Akamatsu S, Takahashi A, Ito M, Ogura K. (2010). Primary signet-ring cell carcinoma of the urinary bladder. Urology 75 (3): $615-8$.

[9] Wang J, Wang FW, Kessinger A. (2012). The impact of signet-ring cell carcinoma histology on bladder cancer outcome. World journal of urology 30 (6): 777-83.

[10] Kinra P, Rashmi SP, Alam A, Singh H, Dash SC. (2017). Primary signet cell adenocarcinoma of bladder. Indian journal of pathology \& microbiology 60 (4): 584-6.

[11] Chiesa-Vottero A. (2020). CDX2, SATB2, GATA3, TTF1, and PAX8 Immunohistochemistry in Krukenberg Tumors. International journal of gynecological pathology : official journal of the International Society of Gynecological Pathologists 39 (2): 170-7.

[12] Yang C, Sun L, Zhang L, Zhou L, Zhao M, Peng Y, et al. (2018). Diagnostic Utility of SATB2 in Metastatic Krukenberg Tumors of the Ovary: An Immunohistochemical Study of 70 Cases With Comparison to CDX2, CK7, CK20, Chromogranin, and Synaptophysin. 42 (2): 160-71.

[13] Lendorf M, Dohn L, Á Dunga B, Loya A, Pappot HJSjou. (2018). An updated review on primary signet-ring cell carcinoma of the urinary bladder and report of a case. 52 (2): 87-93.

[14] Jue JS, Koru-Sengul T, Moore KJ, Miao F, Alameddine M, Nahar B, et al. (2018). Sociodemographic and survival disparities for histologic variants of bladder cancer. The Canadian journal of urology 25 (1): 9179-85.

[15] Jayarajah U, Fernando DMH, Herath KB, de Silva MVC, Goonewardena SAS. (2017). Primary Signet-Ring Cell Adenocarcinoma of the Urinary Bladder Treated with Partial Cystectomy: A Case Report and Review of the Literature. Case reports in urology 2017: 6829692.

[16] Hamakawa T, Kojima Y, Naiki T, Kubota Y, Yasui T, Tozawa K, et al. (2013). Long-term survival of a patient with invasive signet-ring cell carcinoma of the urinary bladder managed by combined s-1 and Cisplatin adjuvant chemotherapy. Case reports in urology 2013: 915874. 